\section{The Use of a Dyeline Photocopier in a Hospital Laboratory}

\section{R. L. McCORRY}

\section{From the Tyrone County Hospital Laboratory, Omagh}

(RECEIVED FOR PUBLICATION MARCH 17, 1960)

New systems for recording results in hospital laboratories have been described by Maxwell (1957) and by Brown, Gillespie, McGowan, and Raper (1959). In their systems the form received with the specimen is a two-part combined request-report form with carbon paper interleaved. In the blank report section, the laboratory stamps a proforma. So far as possible results are recorded directly on the form; the original is returned to the ward and the carbon duplicate is retained in the laboratory.

Photocopying is an alternative to carbon paper for making duplicate copies. Of the methods available (Cffice Magazine, 1958) we have found the dyeline system simple, rapid, and economical. This system has been used mainly by architects for copying plans ; in recent years small continuous-feed machines have been produced for office use. The copy paper is coated with a diazo compound which is bleached by light waves in the blue region and which darkens when treated with a suitable coupling solution. The copy paper may be safely handled in full daylight. Documents printed on translucent but not necessarily transparent paper may be used as negatives to produce positive copies.
In this laboratory single-part request-report forms are used which are essentially similar in layout to those described by Brown et al. (1959). The forms are printed on paper (Wiggins Teape penetrator) specially designed for use with dyeline systems; it has the appearance of a medium-weight typing paper. Proformas are printed with embossed metal plates in an "addressograph-multigraph" 500 machine (Maxwell, 1957 ; Brown et al., 1959). Where possible results are recorded directly upon the form. The completed form is copied in an Ilford " azoflex 105 " ? dyeline photocopier; the original is returned to the $\vec{\omega}$ ward. In this continuous-feed machine, forms can $\rightarrow$ be copied at the rate of four per minute; a single $\omega_{\sigma}$ copy takes 20-25 seconds. Copy paper costs less $\sigma$ than $\frac{1}{2} \mathrm{~d}$., the form itself a little more than $\frac{1}{2} \mathrm{~d}$. The 을 machine saves a considerable amount of the time of the laboratory secretary ; it can be and is used by any member of the laboratory staff.

My thanks are due to the medical, nursing, and laboratory staff of this hospital for their co-operation; to the Northern Ireland Hospitals Authority for a grant for equipment, and to Mr. Thompson and Mr. Burge of the $\mathbf{O}$ and $M$ department for advice.

\section{REFERENCES}

Brown, N. J., Gillespie, W. A., McGowan, G. K., and Raper, A. B. (1959). Symposium on Hospital Laboratory Records. The Association of Clinical Pathologists, 63rd General Meeting, London.

Maxwell, I. (1957). Amer. .J. clin. Path., 28, 509.

Office Magazine (1958). 5, 616. ["Photocopying: Which Method and Why."] 